\title{
Protein tyrosine phosphatase receptor-like genes are frequently hypermethylated in sporadic colorectal cancer
}

\author{
Izabela Laczmanska ${ }^{1}$, Pawel Karpinski ${ }^{1}$, Marek Bebenek ${ }^{2}$, Tomasz Sedziak ${ }^{2}$, David Ramsey ${ }^{3}$, Elzbieta Szmida ${ }^{1}$ \\ and Maria M Sasiadek ${ }^{1}$
}

The activity of phosphatases could be influenced by genetic, as well as epigenetic alterations. In our study, we have investigated the methylation status of four PTPRs: PTPRM, PTPRT, PTPRR and PTPRZ1, which were pre-selected using microarray techniques as being alternatively methylated in sporadic colorectal cancer (CRC). The analyses were carried out on 131 surgical specimens obtained from sporadic CRC patients. The methylation status of the four genes was examined using methyl specific PCR (MSP). The analysis of promoter methylation using an Illumina $27 \mathrm{~K}$ microarray revealed four protein tyrosine phosphatases PTPRM, PTPRT, PTPRR and PTPRZ1 as being hypermethylated with $\beta$-value $\geqslant 0.2$ and $P \leqslant 0.05$. Subsequent analysis using MSP confirmed these observations - the frequency of promoter methylation was significantly higher in tumor cells compared with matched normal tissue for each of the analyzed genes. There was no association observed between the methylation status of PTPRs and either CIMP, K-ras (codon 12) and BRAF (exon 15, V600E) mutations or tumor localization (proximal/distal). The results of our study show a statistically significant difference between promoter methylation in cancerous and healthy tissue. This result supports the hypothesis that the PTPR family has an important role in the etiology of CRC.

Journal of Human Genetics (2013) 58, 11-15; doi:10.1038/jhg.2012.119; published online 25 October 2012

Keywords: colorectal cancer; methylation; protein tyrosine phosphatase receptor-like; PTPRM; PTPRT; PTPRR; PTPRZ1

\section{INTRODUCTION}

Via dephosphorylation of multiple enzymes, protein tyrosine phosphatases (PTPs) regulate many processes, such as cell differentiation and proliferation, apoptosis, gene transcription, mRNA processing, molecule transport out of and into cells, as well as cell signaling.., 2 The PTP Cys-based superfamily is composed of three classes: Class I (classical PTPs) consisting of transmembrane receptor PTPs, nonreceptor PTPs, as well as dual-specific PTPs; Classes II and III consisting of Cdc25 family members and low molecular weight phosphatases, respectively. ${ }^{1}$

PTPs have an important role in carcinogenesis acting as tumor suppressor genes. ${ }^{1-3}$

Loss of the tumor suppressor function of PTPs, similar to that of other tumor suppressors, is a consequence of gene inactivation resulting from a biallelic inactivation event, such as gene deletion, mutation or promoter hypermethylation. ${ }^{4}$ Methylation of $\mathrm{CpG}$ islands located in a gene promoter is associated with the downregulation of gene transcription through the alteration of chromatin conformation influencing DNA availability to transcription factors. ${ }^{5-7}$
Until now, several phosphatases have been examined in human cancers, enabling the assessment of their contribution to the development of cancer. Somatic mutations have been found in the following six phosphatases: PTPRF, PTPRG, PTPRT, PTPN3, PTPN13 and PTPN14 in colorectal cancer (CRC). Epigenetic silencing was observed for PTPRR and PTPRG in CRC and cutaneous T-cell lymphoma, for $P T P R D$ in glioblastoma, head and neck squamous cell carcinoma and lung cancer, as well as for PTPRO in hepatocellular carcinoma. Moreover, loss of heterozygosity was identified for PTPRJ in CRC. ,, $8-12^{2}$

The purpose of our study was to analyze the methylation status of CRC tissues. Using a genome-wide approach (Illumina Infinium HumanMethylation27), we found 422 differentially methylated probes including four members of the tyrosine phosphatase family (PTPRs): PTPRT, PTPRR, PTPRM and PTPRZ1.

These four, but no other PTPs, were selected as they were found to be alternatively methylated by a microarray experiment.The results were then validated using methyl specific PCR (MSP). The tumor suppressor function of PTPs allowed us to hypothesize that epigenetic silencing is a possible mechanism adding to the loss of functioning of these genes in colon cancer cells.

\footnotetext{
${ }^{1}$ Department of Genetics, Wroclaw Medical University, Wroclaw, Poland; ${ }^{2} 1$ st Department of Surgical Oncology, Lower Silesian Oncology Center, Wroclaw, Poland and ${ }^{3}$ Department of Mathematics and Statistics, University of Limerick, Limerick, Ireland 


\section{MATERIALS AND METHODS}

\section{Patient samples}

The study was performed on 131 sporadic CRC samples compared with matched healthy colon tissues. The samples were obtained immediately after surgery from the First Department of Surgical Oncology, Lower Silesian Oncology Centre in Wroclaw. The study group consisted of 59 females and 72 males with mean age $64.5 \pm 10.54$ diagnosed with primary sporadic CRC. None of them were treated with preoperative therapy. The molecular characteristics of the cancer samples are as follows: the BRAF V600E mutation (exon 15) was observed in 8, while the $K$-ras (codon 12) mutation was found in 31 colon cancer samples. No K-ras mutation in codon 13 was revealed in our material. CIMP-high and CIMP-low/0 status were observed for 7 and 124 tumors, respectively.

The study was accepted by Wroclaw Medical University Ethical Committee.

\section{Methods}

The samples were obtained during surgery and were frozen at $-20{ }^{\circ} \mathrm{C}$, until DNA isolation. All tissues were examined histopathologically. DNA was isolated from tumor samples with at least $40 \%$ viable neoplastic cells using a QIAamp DNA mini kit (Qiagen, Hilden, Germany) following the recommended protocol. Methylation status was evaluated for 12 pairs of CRC and matched normal colon tissue using Illumina Infinium HumanMethylation27 (Illumina, San Diego, CA, USA). The Benjamini-Hochberg procedure for controlling the false discovery rate was used to correct $P$-values for multiple testing. All probes with $\beta$-values $\geqslant 0.20$ and $P \leqslant 0.05$ were retained (see Supplementary Table 1 for the detailed results). Further analysis on an additional 131 samples considered four PTP receptor-like genes: PTPRM (probeID: cg01946574), PTPRT (probeID: cg13168820), PTPRR (probeID: cg23694248) and PTPRZ1 (probeID: cg25167643), as they showed differential methylation characterized by $\beta$-values $\geqslant 0.20$ and $P \leqslant 0.05$. The methylation status of these genes was confirmed using MSP. Primers for methylated and unmethylated promoter sequences were designed using MethPrimer software ${ }^{13}$ (Table 1).

DNA modification was performed using the EpiTect Bisulfite Kit (Qiagen) according to the standard procedure. One microgram of DNA isolated from both CRC and healthy colon tissue was modified for each patient.

CpG Methylated HELa Genomic DNA (New England Biolabs, Pickering, ON, Canada) and DNA from the peripheral blood of a healthy person were used as control DNA for methylated and unmethylated sequences, respectively.
The PCR mixture was prepared separately for methylated and unmethylated sequences for all the genes examined. Briefly: $0.6 \mu \mathrm{l}$ modified DNA was mixed with $0.75 \mu \mathrm{l}$ of each primer $(10 \mathrm{~mm}) 0.75 \mu \mathrm{l}$ dNTPs $(40 \mathrm{~mm}$, Fermentas, Burlington, ON, Canada), $1.5 \mu \mathrm{l}$ buffer $(10 \times$, Qiagen), $0.15 \mu \mathrm{l}$ HotStart DNA Polymerase $\left(5 \mathrm{u}^{-1} \mathrm{l}^{-1}\right.$ Qiagen) in a total volume of $15 \mu \mathrm{l}$.

The cycle parameters were as follows: $95^{\circ} \mathrm{C} 15 \mathrm{~min}, 95^{\circ} \mathrm{C} 30 \mathrm{~s}, 56^{\circ} \mathrm{C} 30 \mathrm{~s}$ (methylated and unmethylated PTPRM and PTPRZ1), or $60^{\circ} \mathrm{C}$ (methylated and unmethylated PTPRT, unmethylated PTPRR) or $63^{\circ} \mathrm{C}$ (methylated PTPRR) for $35-38$ cycles, $72{ }^{\circ} \mathrm{C} 30 \mathrm{~s}$ and a $72{ }^{\circ} \mathrm{C} 5 \mathrm{~min}$ final extension in an MJ thermocycler. The products were evaluated using $2.5 \%$ agarose gel.

\section{Statistical analysis}

The association between the methylation of PTPRM, PTPRZ1, PTPRT and PTPRR and the type of colon cell (cancerous or normal) was analyzed using Fisher's exact test of association. This test was also used to analyze the association of the methylation status of these four PTPRs with various clinical factors (for example, location of cancer, mutations and CIMP status).

\section{RESULTS}

In this study, we analyzed the methylation status of four PTPs in 131 sporadic colon cancers (30 proximal/101 distal) in comparison to matched healthy colon tissues (95 pairs tumor/healthy tissue and 36 cancer tissues without paired healthy tissue).

\section{Microarray results}

Using genome-wide microarray analysis, we found over 400 differentially methylated genes in tumor tissues, among which four PTP genes were found as follows: PTPRM, PTPRT, PTPRR and PTPRZ1 (see Table 2 and Supplementary Table 1).

\section{MSP results}

Partial methylation (the presence of both methylated and unmethylated sequences), lack of methylation (only unmethylated sequences), as well as complete methylation (only methylated sequences) were observed for the examined phosphatases in cancer tissues (Figure 1) A few samples amplified neither of the two sets of primers. The results are presented in Table 3.

Table 1 Primer sequences for the analyzed genes

\begin{tabular}{|c|c|c|c|c|}
\hline $\begin{array}{l}\text { Gene, localization and no. of nucleotides from } \\
\text { ATG for forward primer with reference sequence }\end{array}$ & $\% G C$ & 'C's & $\begin{array}{l}\text { Primer sequences } F M, R M \text {-forward/reverse, } \\
\text { methylated } F U, R U \text { - forward/ reverse, unmethylated }\end{array}$ & Product size \\
\hline PTPRR & 64.00 & 9 & FM 5'TGGAGTTTATTTTGTTTTAAGTCGT & 197 \\
\hline \multirow[t]{3}{*}{ 12q21.2-440 (NM_002849.3) } & 64.00 & 6 & RM 5'GACAAATACCTAACCTTCTAAACGC & \\
\hline & 64.00 & 9 & FU 5'TGGAGTTTATTTTGTTTTAAGTTGT & 199 \\
\hline & 66.67 & 7 & RU 5'ACAACAAATACCTAACCTTCTAAACAC & \\
\hline PTPRM & 75.00 & 6 & FM 5'TCGGGTTTATACGGGATATC & 220 \\
\hline \multirow[t]{3}{*}{ 18p11.2-1230 (NM_002845.3) } & 65.22 & 6 & RM 5'GAAAAACTACGAACTTCTCTACG & \\
\hline & 73.91 & 7 & FU 5'GTTTTGGGTTTATATGGGATATT & 225 \\
\hline & 68.00 & 6 & RU 5'CCAAAAAACTACAAACTTCTCTACA & \\
\hline PTPRT & 75.00 & 9 & FM 5'TGTTCGAGATTTTGGTTAGTAGTC & 231 \\
\hline \multirow[t]{3}{*}{ 20q12-q13.1-723 (NM_133170.3) } & 73.91 & 8 & RM 5'ACATCGTCTACCCTATAAAAACG & \\
\hline & 76.00 & 9 & FU 5'GTGTTTGAGATTTTGGTTAGTAGTT & 233 \\
\hline & 72.00 & 8 & RU 5'AACATCATCTACCCTATAAAAACAC & \\
\hline PTPRZ1 & 75.00 & 5 & FM 5'AGGTACGTTTTTTAGCGGAGC & 225 \\
\hline \multirow[t]{3}{*}{ 7q31.1-454 (NM_001206839.1) } & 50.00 & 5 & RM 5'ATAATCTAAAAAAAACCGCACGAT & \\
\hline & 78.26 & 5 & FU 5'GAGGTATGTTTTTAGTGGAGTGG & 228 \\
\hline & 50.00 & 6 & RU 5'AAATAATCTAAAAAAAACCACACAAT & \\
\hline
\end{tabular}


In healthy colon tissues, no complete methylation occurred, but cases of partial methylation and lack of methylation were common in the following genes: PTPRM, PTPRT and PTPRZ1 with the exception of PTPRR, for which complete methylation was found in 2 normal samples (Table 3 ).

Our results revealed a significantly higher level of methylation in tumors compared with normal colon tissue (see Table 4).

There was no significant association of the methylation status of PTPRM, PTPRT, PTPRR or PTPRZ1 with CIMP, the K-ras (codon 12) and BRAF (exon 15, V600E) mutations, or tumor localization (proximal/distal) (data not shown).

A clear association between the methylation of PTPRZ1 and PTPRM in healthy cells $(P<0.001)$ was also found (Table 5).

\section{DISCUSSION}

An abnormal methylation pattern is a widely described biological phenomenon in cancer cells. Global hypomethylation, promoting genomic instability and regional hypermethylation of $\mathrm{CpG}$ islands at

Table 2 Results obtained from an Illumina27K microarray for selected PTPs

\begin{tabular}{lccc}
\hline Gene & OMIM and localization & P-value & Diff. Beta \\
\hline PTPRM & $176888 / 18 \mathrm{p} 11.2$ & 0.00271947 & 0.28216614 \\
PTPRT & $608712 / 20 \mathrm{q} 12-\mathrm{q} 13.1$ & 0.00093595 & 0.29058374 \\
PTPRR & $602853 / 12 \mathrm{q} 21.2$ & 0.04606745 & 0.21622813 \\
PTPRZ1 & $176891 / 7 \mathrm{q} 31.1$ & 0.0485365 & 0.26768926 \\
\hline
\end{tabular}

gene promoters associated with transcriptional silencing of the tumor suppressor gene, has been reported in many types of cancers. ${ }^{6}$ In particular, the hypermethylation of tumor suppressor gene promoters leading to loss of their function is an important event in the molecular pathogenesis of cancer. ${ }^{14}$

Our results revealed that the promoter sequences of four PTPs: PTPRM, PTPRT, PTPRR and PTPRZ1, are hypermethylated in colon cancer compared with healthy colon tissue. These data were obtained using a methylation microarray and then confirmed by methylationspecific PCR. Methylated and unmethylated sequences were both often observed in healthy colon tissues in our study, although the frequency of methylated sequences was significantly lower than in cancer tissues (for PTPRM, PTPRT, PTPRZ1 P=0 and for PTPRR $P=0.02835$ ). However, using MSP only the presence of a methylated allele in the tissue examined could be shown without the possibility of distinguishing between uni- and biallelic methylation.

These observations should be analyzed in the context of the possibility of PTPs having a role in the development of cancer. As published recently, the involvement of PTPs in cancerogenesis has been observed for a variety of tumors. PTPRT $(P T P R-\rho)$ is a known tumor suppressor, mutations of which are associated with melanoma, colon, stomach and lung cancer. ${ }^{3}$ As reported previously, PTPRT mutations may cause loss of its function (nonsense or frameshift mutations), a decrease in phosphatase activity or defective cell adhesion (missense mutations in phosphatase and extracellular domains, respectively). ${ }^{3,8}$ Moreover, an increase in PTPRT activity in a tumor cell culture inhibited cell growth. ${ }^{8}$

PTPRZ1 (PTPR- $\zeta$ or PTPR- $\beta)$ is a receptor of VacA, a cytotoxin of Helicobacter pylori, inducing gastric ulcers. ${ }^{15}$ PTPRZ1 has also been
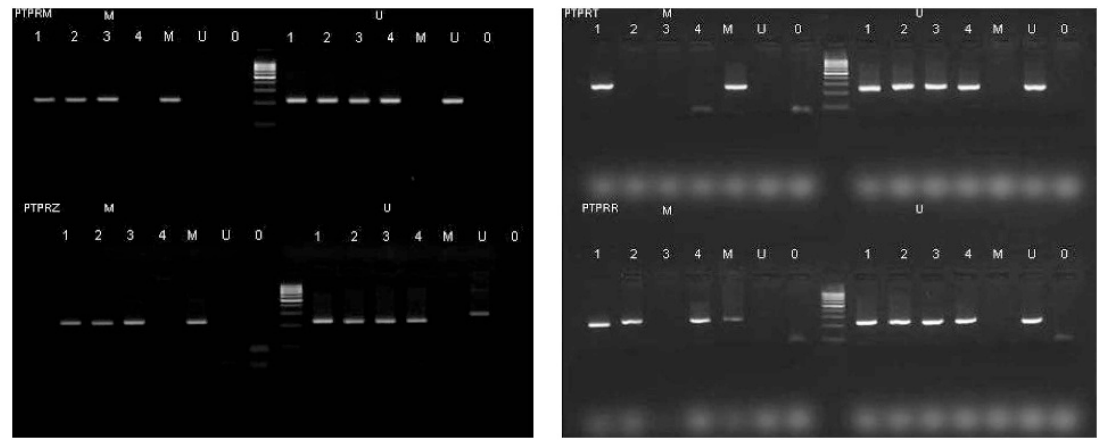

Figure 1 Methylation-specific polymerase chain reaction electrophoresis for cancerous and normal tissues (M—primers for methylated sequence, U—primers for unmethylated sequence) for four analyzed genes: PTPRM (220 and $225 \mathrm{bp}$ ), PTPRZ1 (225 and $228 \mathrm{bp}$ ), PTPRT (231 and $233 \mathrm{bp)}$ and PTPRR (197 and $199 \mathrm{bp})$. Lanes 1 and 2-cancer and matched normal DNA, lanes 3 and 4-cancer and matched normal DNA, M-positive control for methylation, U—positive control for lack of methylation, 0-negative control. Size standard—O'GeneRuler 100 bp DNA Ladder (Thermo Scientific Fermentas, Burlington, ON, Canada).

Table 3 Methylation status in the analyzed tissues

\begin{tabular}{|c|c|c|c|c|c|c|c|c|}
\hline \multirow[b]{2}{*}{ Gene } & \multicolumn{4}{|c|}{ Cancer tissue 131} & \multicolumn{4}{|c|}{ Healthy tissue 95} \\
\hline & $\begin{array}{c}\text { Partial } \\
\text { methylation }\end{array}$ & $\begin{array}{c}\text { Complete } \\
\text { methylation }\end{array}$ & $\begin{array}{c}\text { Lack of } \\
\text { methylation }\end{array}$ & $\begin{array}{c}\text { Lack of both } \\
\text { sequences }\end{array}$ & $\begin{array}{c}\text { Partial } \\
\text { methylation }\end{array}$ & $\begin{array}{c}\text { Complete } \\
\text { methylation }\end{array}$ & $\begin{array}{c}\text { Lack of } \\
\text { methylation }\end{array}$ & $\begin{array}{c}\text { Lack of both } \\
\text { sequences }\end{array}$ \\
\hline PTPRM & 122 (93.1\%) & $1(0.8 \%)$ & $7(5.3 \%)$ & $1(0.8 \%)$ & $54(56.8 \%)$ & 0 & $41(43.2 \%)$ & 0 \\
\hline PTPRT & 104 (79.4\%) & $4(3.1 \%)$ & $21(16.0 \%)$ & $2(1.5 \%)$ & 0 & 0 & $95(100 \%)$ & 0 \\
\hline PTPRR & 101 (77.1\%) & $5(3.8 \%)$ & $22(16.8 \%)$ & $3(2.3 \%)$ & $62(65.3 \%)$ & $2(2.1 \%)$ & $31(32.6 \%)$ & 0 \\
\hline PTPRZ1 & 123 (93.9\%) & $7(5.3 \%)$ & 0 & $1(0.8 \%)$ & $49(51.6 \%)$ & 0 & $46(48.4 \%)$ & 0 \\
\hline
\end{tabular}


Table 4 Number of samples with methylation in the promoter sequence for the PTPs examined

\begin{tabular}{lccc}
\hline Gene & Cancer tissue 131 & Healthy tissue 95 & P-value \\
\hline PTPRM & $123(93.9 \%)$ & $54(56.8 \%)$ & 0 \\
PTPRT & $108(82.4 \%)$ & $0(100 \%)$ & 0 \\
PTPRR & $106(80.9 \%)$ & $64(67.4 \%)$ & 0.02835 \\
PTPRZ1 & $130(99.23 \%)$ & $49(51.6 \%)$ & 0 \\
\hline
\end{tabular}

Abbreviation: PTP, protein tyrosine phosphatase.

Table 5 Association between methylation of PTPRZ1 and PTPRM in healthy cells $P<0.001$

\begin{tabular}{lcc}
\hline & $\begin{array}{c}\text { Methylation not } \\
\text { observed at PTPRM }\end{array}$ & $\begin{array}{c}\text { Methylation } \\
\text { observed at PTPRM }\end{array}$ \\
\hline Methylation not observed at PTPRZ1 & 29 & 17 \\
Methylation observed at PTPRZ1 & 12 & 39 \\
\hline
\end{tabular}

reported to have an important role in the functioning of neurons. ${ }^{15}$ There are no reports on its role as a tumor suppressor or its significance for cell homeostasis.

PTPRM $(P T P R-\mu)$ is a member of the Ig superfamily of adhesion molecules and has been shown to be expressed in neurons, glia, epithelia and prostate. ${ }^{16-18}$ PTPRM has a crucial role in epidermal growth factor receptor signaling-reduced expression of PTPRM increases the epidermal growth factor receptor specific phosphorylation of tyrosine residue, thus causing phospholipase C-gamma (PLC) $\gamma_{1}$ activation and cell migration. ${ }^{18}$ It is also known that epidermal growth factor receptor activation via phosphorylation can itself increase cell proliferation, differentiation and survival. ${ }^{18}$ The increased expression of PTPRM in normal cell and low-grade astrocytomas compared with high-grade glioblastomas, along with the results of in vivo experiments, also confirmed that loss of this protein promotes tumor cell migration and propagation. ${ }^{17}$ The lack of expression of PTPRM in prostate cancer cells connected with the lack of PTPRM-mediated adhesion also suggests a role of PTPRM as a tumor suppressor gene. ${ }^{16}$

PTPRR was firstly described by Menigatti et al. ${ }^{9}$ to be highly downregulated in the early stages of colorectal tumorigenesis. This gene encoding a classical PTP receptor has a crucial role in homeostasis regulating signaling in proliferation and differentiation.

PTPRT was the only gene for which there was no methylated sequence observed in the control samples. The importance of PTPRT in colon cancer has been postulated by Wang et al. ${ }^{8}$ Our results support this hypothesis, showing that the most probable role of PTPRT is the suppression of carcinogenesis. However, further studies are needed to confirm this hypothesis.

We also found a clear association between the methylation of PTPRZ1 and PTPRM in healthy cells $(P<0.001)$. Up to now, no data suggesting any biological interaction between these genes have been published. PTPRM is known to have a crucial role in the epidermal growth factor receptor signaling pathway and thus in cell migration, while the role of PTPRZ1 is still poorly described. Up to now, the expression of PTPRZ1 has only been shown in neurons. Our results are the first revealing a potential role of this gene in colon cells.

Recent epigenomic microarray analyses in CRC tissues have identified many gene promoters, which were hypermethylated. Thus, it has been postulated that these genes may be involved in colorectal tumorigenesis. In the studies of Kim et al. ${ }^{19}$ and Hinoue et al., ${ }^{20}$ the Illumina platform has been used to analyze 22 and 125 tumor samples, respectively. Although these authors have not mentioned PTPs in the 'top ranking lists' of genes with aberrant methylation, the available raw data show differences in PTP methylation compared with healthy tissue. ${ }^{19,20}$

Our study, which seems to be the first analysis using MSP of the methylation status of PTPRs in sporadic colon cancer, has enabled us to conclude that the methylation of PTPRs is significantly different in cancerous and healthy tissue. This suggests a potential role of these genes as suppressors in colorectal carcinogenesis. Moreover, our results revealing alterations in PTPRZ1 methylation in normal colon samples led us to hypothesize that this gene has a still unknown role in the colon.

\section{ACKNOWLEDGEMENTS}

This paper has been supported by the State Committee for Scientific Research, Polish Ministry for Scientific Research and Information Technology (grant no. N N401 601438), 2010-2013 and by a research fellowship within the 'Development Program of Wroclaw Medical University' funded by the European Social Fund, Human Capital, National Cohesion Strategy '(contract no. UDA-POKL 04.01.01-00-010/08-00)'. David Ramsey is supported by Science Foundation Ireland under the BIO-SI project (no. 07MI012).

1 Alonso, A., Sasin, J., Bottini, N., Friedberg, I., Friedberg, I., Osterman, A. et al. Protein tyrosine phosphatases in the human genome. Cell 117, 699-711 (2004).

2 Arena, S., Benvenuti, S. \& Bardelli, A. Genetic analysis of the kinome and phosphatome in cancer. Cell Mol. Life Sci. 62, 2092-2099 (2005).

3 Scott, A. \& Wang, Z. Tumour suppressor function of protein tyrosine phosphatase receptor-T. Biosci. Rep. 31, 303-307 (2011).

4 Ponder, B. A. Cancer genetics. Nature 411, 336-341 (2001)

5 van Doorn, R., Zoutman, W. H., Dijkman, R., de Menezes, R. X., Commandeur, S., Mulder, A. A. et al. Epigenetic profiling of cutaneous T-cell lymphoma: promoter hypermethylation of multiple tumor suppressor genes including BCL7a, PTPRG, and p73. J. Clin. Oncol. 23, 3886-3896 (2005).

6 Sharma, S., Kelly, T. K. \& Jones, P. A. Epigenetics in cancer. Carcinogenesis 31, 27-36 (2010).

7 Illingworth, R., Kerr, A., Desousa, D., Jørgensen, H., Ellis, P., Stalker, J. et al. A novel CpG island set identifies tissue-specific methylation at developmental gene loci. PLoS Biol. 6, e22 (2008).

8 Wang, Z., Shen, D., Parsons, D. W., Bardelli, A., Sager, J., Szabo, S. et al. Mutationa analysis of the tyrosine phosphatome in colorectal cancers. Science 304, 1164-1166 (2004).

9 Menigatti, M., Cattaneo, E., Sabates-Bellver, J., Ilinsky, V. V., Went, P., Buffoli, F. et al. The protein tyrosine phosphatase receptor type $\mathrm{R}$ gene is an early and frequent target of silencing in human colorectal tumorigenesis. Mol. Cancer 8, 124 (2009).

10 van Roon, E. H., de Miranda, N. F., van Nieuwenhuizen, M. P., de Meijer, E. J., van Puijenbroek, M., Yan, P. S. et al. Tumour-specific methylation of PTPRG intron 1 locus in sporadic and Lynch syndrome colorectal cancer. Eur. J. Hum. Genet. 19, 307-312 (2011)

11 Motiwala, T., Ghoshal, K., Das, A., Majumder, S., Weichenhan, D., Wu, Y. Z. et al. Suppression of the protein tyrosine phosphatase receptor type 0 gene (PTPRO) by methylation in hepatocellular carcinomas. Oncogene 22, 6319-6331 (2003)

12 Veeriah, S., Brennan, C., Meng, S., Singh, B., Fagin, J. A., Solit, D. B. et al. The tyrosine phosphatase PTPRD is a tumor suppressor that is frequently inactivated and mutated in glioblastoma and other human cancers. Proc. Natl Acad. Sci. USA 106, 9435-9440 (2009)

$13 \mathrm{Li}, \quad$ L. C. \& Dahiya, R. MethPrimer: designing primers for methylation PCRs Bioinformatics 18, 1427-1431 (2002)

14 Boultwood, J. \& Wainscoat, J. S. Gene silencing by DNA methylation in haematological malignancies. Br. J. Haematol. 138, 3-11 (2007).

15 Fujikawa, A., Fukada, M., Makioka, Y., Suzuki, R., Chow, J. P., Matsumoto, M. et al. Consensus substrate sequence for protein-tyrosine phosphatase receptor type $Z$. J. Biol. Chem. 286, 37137-37146 (2011).

16 Hellberg, C. B., Burden-Gulley, S. M., Pietz, G. E. \& Brady-Kalnay, S. M. Expression of the receptor protein-tyrosine phosphatase, PTPmu, restores E-cadherin-dependent adhesion in human prostate carcinoma cells. J. Biol. Chem. 277, 11165-11173 (2002) 
17 Burgoyne, A. M., Palomo, J. M., Phillips-Mason, P. J., Burden-Gulley, S. M., Major, D. L., Zaremba, A. et al. PTPmu suppresses glioma cell migration and dispersal. Neuro. Oncol. 11, 767-778 (2009).

18 Hyun, S. W., Anglin, I. E., Liu, A., Yang, S., Sorkin, J. D., Lillehoj, E. et al. Diverse injurious stimuli reduce protein tyrosine phosphatase- $\mu$ expression and enhance epidermal growth factor receptor signaling in human airway epithelia. Exp. Lung. Res. 37, 327-343 (2011).
19 Kim, Y. H., Lee, H. C., Kim, S. Y., Yeom, Y. I., Ryu, K. J., Min, B. H. et al. Epigenomic analysis of aberrantly methylated genes in colorectal cancer identifies genes commonly affected by epigenetic alterations. Ann. Surg. Oncol. 18, 2338-2347 (2011).

20 Hinoue, T., Weisenberger, D. J., Lange, C. P., Shen, H., Byun, H. M., Van Den Berg, D. et al. Genome-scale analysis of aberrant DNA methylation in colorectal cancer. Genome Res. 22, 271-282 (2012).

Supplementary Information accompanies the paper on Journal of Human Genetics website (http://www.nature.com/jhg) 